Discussiones Mathematicae

Graph Theory 22 (2002) 7-15

\title{
HEAVY CYCLES IN WEIGHTED GRAPHS
}

\author{
J. ADRIAN BONDY* \\ Laboratoire de Mathématiques Discrètes \\ Université Claude \\ Bernard Lyon 1, 69622 Villeurbanne Cedex, France \\ Hajo J. Broersma \\ Faculty of Applied Mathematics \\ University of Twente \\ P.O. Box 217, 7500 AE Enschede, The Netherlands \\ JAN VAN DEN HEUVEL ${ }^{* \dagger}$ \\ Department of Mathematics and Statistics \\ Simon Fraser University \\ Burnaby, B.C., Canada V5A $1 S 6$ \\ AND \\ HenK JAN Veldman \\ Faculty of Applied Mathematics \\ University of Twente \\ P.O. Box 217, 7500 AE Enschede, The Netherlands
}

The first three authors would like to dedicate this paper to Henk Jan Veldman, a valued colleague and beloved friend who died October 12, 1998.

${ }^{*}$ Visits to the University of Twente supported by the Euler Institute for Discrete Mathematics and its Applications.

${ }^{\dagger}$ Supported by the Natural Sciences and Engineering Research Council of Canada, International Fellowship. Current address: Centre for Discrete and Applicable Mathematics, Department of Mathematics, London School of Economics, Houghton Street, London WC2A 2AE, U.K. 
8 J.A. Bondy, H.J. Broersma, J. van den Heuvel, H.J. Veldman

\begin{abstract}
An (edge-)weighted graph is a graph in which each edge $e$ is assigned a nonnegative real number $w(e)$, called the weight of $e$. The weight of a cycle is the sum of the weights of its edges, and an optimal cycle is one of maximum weight. The weighted degree $w(v)$ of a vertex $v$ is the sum of the weights of the edges incident with $v$. The following weighted analogue (and generalization) of a well-known result by Dirac for unweighted graphs is due to Bondy and Fan. Let $G$ be a 2-connected weighted graph such that $w(v) \geq r$ for every vertex $v$ of $G$. Then either $G$ contains a cycle of weight at least $2 r$ or every optimal cycle of $G$ is a Hamilton cycle. We prove the following weighted analogue of a generalization of Dirac's result that was first proved by Pósa. Let $G$ be a 2-connected weighted graph such that $w(u)+w(v) \geq s$ for every pair of nonadjacent vertices $u$ and $v$. Then $G$ contains either a cycle of weight at least $s$ or a Hamilton cycle. Examples show that the second conclusion cannot be replaced by the stronger second conclusion from the result of Bondy and Fan. However, we characterize a natural class of edge-weightings for which these two conclusions are equivalent, and show that such edge-weightings can be recognized in time linear in the number of edges.
\end{abstract}

Keywords: weighted graph, (long, optimal, Hamilton) cycle, (edge-, vertex-)weighting, weighted degree.

2000 Mathematics Subject Classifications: 05C45, 05C38, 05C35.

\title{
1. Introduction
}

We use [6] for terminology and notation not defined here and consider finite simple graphs only.

By an edge-weighting of a graph $G=(V, E)$ we shall mean a function $w: E \rightarrow \mathbb{R}$ such that $w(e)$, the weight of $e$, is nonnegative for all $e \in E$. A graph provided with an edge-weighting is called an edge-weighted graph or simply a weighted graph. The weight of a subgraph $H$ of $G$ is defined by

$$
w(H)=\sum_{e \in E(H)} w(e) .
$$

An optimal cycle of $G$ is a cycle of maximum weight. The weighted degree $w(v)$ of a vertex $v$ of $G$ is the sum of the weights of the edges incident with $v$.

A well-known result due to Dirac [7] is the following. 
Theorem 1 [7]. Let $G$ be a 2-connected graph such that $d(v) \geq r$ for every vertex $v$ of $G$. Then $G$ contains either a cycle of length at least $2 r$ or a Hamilton cycle.

The following generalization of Theorem 1 is contained implicitly in Pósa [8].

Theorem 2 [8]. Let $G$ be a 2-connected graph such that $d(u)+d(v) \geq s$ for every pair of nonadjacent vertices $u$ and $v$. Then $G$ contains either a cycle of length at least $s$ or a Hamilton cycle.

An unweighted graph can be regarded as a weighted graph in which each edge has weight 1 . Thus, in an unweighted graph, $w(v)=d(v)$ for each vertex $v$, and an optimal cycle is simply a longest cycle. Hence the following result from [3] generalizes Theorem 1.

Theorem 3 [3]. Let $G$ be a 2-connected weighted graph such that $w(v) \geq r$ for every vertex $v$ of $G$. Then either $G$ contains a cycle of weight at least $2 r$ or every optimal cycle of $G$ is a Hamilton cycle.

Note that Theorem 3 does not remain valid if negative weights are allowed. To see this, subdivide every edge of $K_{2,3} k \geq 1$ times and assign weight -1 to every edge of the resulting graph.

In [3] the natural question was asked whether Theorem 2 admits an analogous generalization, strengthening Theorem 3 .

Question A [3]. Let $G$ be a 2-connected weighted graph such that $w(u)+$ $w(v) \geq s$ for every pair of nonadjacent vertices $u$ and $v$. Is it true that either $G$ contains a cycle of weight at least $s$ or every optimal cycle is a Hamilton cycle?

It is perhaps surprising that the answer to Question A is negative; a counterexample was first provided by Yan Lirong [10]. For complete graphs, Question A amounts to the following.

Question $\mathbf{A}^{\prime}$. Is every optimal cycle in a weighted complete graph a Hamilton cycle?

Even this is not true. For $n \geq 4$, let $K_{n}(s)$ denote the weighted complete graph of order $n$ obtained by assigning weight 1 to the edges incident with one fixed vertex and weight $s$ to all other edges. Only if $s<2$ is every 
optimal cycle a Hamilton cycle. For $s=2$ there exists an optimal cycle which is not a Hamilton cycle, while for $s>2$ no optimal cycle is a Hamilton cycle. Note that in Theorem 3, applied with $r=n-1$ to $K_{n}(s)$, the first conclusion holds for $s \geq 2$ while the alternative conclusion holds for $s<2$.

Despite this negative answer to Question $\mathrm{A}^{\prime}$ (and a fortiori to Question A), Theorem 2 does indeed admit a generalization to weighted graphs, as follows.

Theorem 4. Let $G$ be a 2-connected weighted graph such that $w(u)+w(v) \geq$ $s$ for every pair of nonadjacent vertices $u$ and $v$. Then $G$ contains either a cycle of weight at least $s$ or a Hamilton cycle.

This will be proved in Section 2. Theorem 4 does not generalize Theorem 3, since the second conclusion in Theorem 3 is stronger than the corresponding one in Theorem 4. However, there is a natural class of edge-weightings for which the second conclusions in Theorems 3 and 4 are equivalent, namely the positive-induced edge-weightings defined next.

Let $w$ be an edge-weighting of a graph $G=(V, E)$ and assume there exists a function $w^{\prime}: V \rightarrow \mathbb{R}$ (a vertex-weighting of $G$ ) such that, for every edge $u v$ of $G$,

$$
w(u v)=\frac{w^{\prime}(u)+w^{\prime}(v)}{2} .
$$

Then we say that the edge-weighting $w$ is induced (by the vertex-weighting $\left.w^{\prime}\right)$. If $w^{\prime}$ can be chosen in such a way that $w^{\prime}(v)>0$ for all $v \in V$, then we call $w$ positive-induced. For every cycle $C$ of $G$ we have

$$
w(C)=\sum_{e \in E(C)} w(e)=\sum_{v \in V(C)} w^{\prime}(v) .
$$

Hence if $w$ is positive-induced, the statements "every optimal cycle of $G$ is a Hamilton cycle" and " $G$ contains a Hamilton cycle" are indeed equivalent.

In a similar vein we can define an edge-weighting $w$ to be nonnegativeinduced if $w$ is induced by a vertex-weighting $w^{\prime}$ such that $w^{\prime}(v) \geq 0$ for all $v \in V$. It follows easily that if $w$ is nonnegative-induced, then the statements "G contains a Hamilton cycle" and "there exists an optimal cycle that is a Hamilton cycle" are equivalent, but neither of these statements implies that "every optimal cycle of $G$ is a Hamilton cycle".

Note that the edge-weighting of $K_{n}(s)$ is induced for all $s$, positiveinduced for $s<2$, and nonnegative-induced for $s \leq 2$. Induced, positiveinduced and nonnegative-induced edge-weightings are characterized in Section 3. 
It is natural to seek weighted generalizations of other extremal theorems, not only for graphs but also for directed graphs. Indeed, the simple observation that a strict directed graph of minimum outdegree $d$ contains a directed path of length $d$ was generalized to weighted directed graphs by Bollobás and Scott [1]. However, no weighted generalization is known of the equally trivial fact that such a directed graph contains a directed cycle of length at least $d+1$. Moreover, examples due to Spencer [9] and, independently, Bollobás and Scott [1] show that quite restrictive edge-weightings will be needed for this. It may be that a directed version of the induced edgeweightings defined here will permit such a generalization.

\section{Proof of Theorem 4}

The proof of the following lemma is implicit in the proof of [4, Lemma 2.1].

Lemma 5. Let $G$ be a 2-connected weighted graph and $P=v_{1} v_{2} \cdots v_{p}$ a path in $G(p \geq 3)$. Define $S=\left\{v_{i} \mid v_{1} v_{i} \in E(G)\right\}$ and $T=\left\{v_{i} \mid v_{i-1} v_{p} \in E(G)\right\}$. Assume each of the following conditions is satisfied.

(a) $\quad N_{G}\left(v_{1}\right) \backslash V(P)=N_{G}\left(v_{p}\right) \backslash V(P)=\varnothing$;

(b) $S \cap T=\varnothing$ (in particular, $\left.v_{1} v_{p} \notin E(G)\right)$;

(c) $\quad w\left(v_{i-1} v_{i}\right) \geq\left\{\begin{aligned} w\left(v_{1} v_{i}\right), & \text { for all } v_{i} \in S ; \\ w\left(v_{i-1} v_{p}\right), & \text { for all } v_{i} \in T \text {. }\end{aligned}\right.$

Then there is a cycle $C$ in $G$ of weight $w(C) \geq w\left(v_{1}\right)+w\left(v_{p}\right)$.

Proof of Theorem 4. Let $G$ be a 2-connected weighted graph such that $w(u)+w(v) \geq s$ for every pair $u, v$ of nonadjacent vertices. Suppose $G$ does not contain a Hamilton cycle. We need to prove that $G$ contains a cycle of weight at least $s$.

Choose a path $P=v_{1} v_{2} \cdots v_{p}$ in $G$ such that

(i) $P$ is as long as possible (i.e., $p$ is maximum);

(ii) $w(P)$ is as large as possible, subject to (i).

Claim. There exists no cycle in $G$ containing all vertices of $P$.

Proof. Suppose there exists a cycle $C$ containing all vertices of $P$. If $V(C) \backslash V(P) \neq \varnothing$, then removing one edge from $C$ gives a path longer than $P$, 
contradicting the choice in (i). So we have $V(C)=V(P)$. Since $C$ is not a Hamilton cycle and $G$ is connected, we can find a vertex $u \in V(G) \backslash V(C)$ and a path $Q$ from $u$ to $v_{j}$ for some $v_{j} \in V(C)$, such that $Q$ is internallydisjoint from $C$. The subgraph $C \cup Q$ of $G$ contains a path longer than $P$, again contradicting the choice in (i).

Define $S$ and $T$ as in Lemma 5 . We complete the proof by showing that Lemma 5 applies, i.e., (a), (b), (c) in Lemma 5 are all satisfied.

By the choice in (i) we immediately see that (a) holds.

To prove (b), suppose $v_{i} \in S \cap T$. Then we can form the cycle $C=$ $v_{1} v_{i} v_{i+1} \cdots v_{p} v_{i-1} v_{i-2} \cdots v_{1}$, which contradicts the Claim. Hence (b) holds.

If $v_{i} \in S$, then the path $P^{\prime}=v_{i-1} v_{i-2} \cdots v_{1} v_{i} v_{i+1} \cdots v_{p}$ has the same length as $P$. So because of (ii), we must have $w(P) \geq w\left(P^{\prime}\right)$, hence $w\left(v_{i-1} v_{i}\right) \geq w\left(v_{1} v_{i}\right)$. Similarly, if $v_{i} \in T, w\left(v_{i-1} v_{i}\right) \geq w\left(v_{i-1} v_{p}\right)$. Thus (c) holds.

\section{Induced Edge-Weightings}

Let $G=(V, E)$ be a graph with an edge-weighting $w$. From the definition, $w$ is induced if and only if we can find a solution of the following set of $|E|$ linear equations in the $|V|$ unknowns $w^{\prime}(v), v \in V$ :

$$
w^{\prime}(x)+w^{\prime}(y)=2 w(x y), \quad \text { for all } x y \in E .
$$

A walk in a graph is an alternating sequence $v_{0} e_{1} v_{1} e_{2} v_{2} \cdots e_{k} v_{k}$ of vertices and edges such that $e_{i}=v_{i-1} v_{i}$, for $i=1, \ldots, k$. The walk is called closed if $v_{0}=v_{k}$. If $W=v_{0} e_{1} v_{1} e_{2} v_{2} \cdots e_{k} v_{k}$ is a walk in $G$, then we define

$$
\phi_{w}(W)=\sum_{i=1}^{k}(-1)^{i-1} w\left(e_{i}\right) .
$$

The following theorem gives a characterization of induced edge-weightings.

Theorem 6. Let $G=(V, E)$ be a graph with an edge-weighting $w$. Then $w$ is induced if and only if for every closed walk $W$ of even length we have $\phi_{w}(W)=0$.

Proof. Without loss of generality we may assume that $G$ is connected. First suppose that $w$ is induced by $w^{\prime}$. Then by (1) we have for every closed walk $W=v_{0} e_{1} v_{1} \cdots e_{2 k} v_{2 k}\left(=v_{0}\right)$ of even length: 


$$
\begin{aligned}
& \phi_{w}(W) \\
& =w\left(e_{1}\right)-w\left(e_{2}\right)+w\left(e_{3}\right)-\cdots-w\left(e_{2 k}\right) \\
& =\frac{1}{2}\left(w^{\prime}\left(v_{0}\right)+w^{\prime}\left(v_{1}\right)\right)-\frac{1}{2}\left(w^{\prime}\left(v_{1}\right)+w^{\prime}\left(v_{2}\right)\right)+\cdots-\frac{1}{2}\left(w^{\prime}\left(v_{2 k-1}\right)+w^{\prime}\left(v_{2 k}\right)\right) \\
& =\frac{1}{2} w^{\prime}\left(v_{0}\right)-\frac{1}{2} w^{\prime}\left(v_{2 k}\right)=0,
\end{aligned}
$$

which proves one side of the theorem.

Now suppose that $\phi_{w}(W)=0$ for every closed walk $W$ of even length. If $G$ is bipartite, choose a vertex $u \in V$ and a real number $a$ and set $w^{\prime}(u)=$ $a$. If $G$ is not bipartite, choose a cycle $C=u_{0} e_{1} u_{1} \cdots e_{2 k-1} u_{0}$ of odd length in $G$, and set $u=u_{0}$ and $w^{\prime}(u)=\phi_{w}(C)$. Now for any vertex $v$, consider a walk from $v$ to $u, W=v f_{1} v_{1} \cdots f_{\ell} u$, and set

$$
w^{\prime}(v)= \begin{cases}2 \phi_{w}(W)+w^{\prime}(u), & \text { if } \ell \text { is even, } \\ 2 \phi_{w}(W)-w^{\prime}(u), & \text { if } \ell \text { is odd }\end{cases}
$$

We claim that this definition of $w^{\prime}(v)$ is independent of the choice of the walk $W$. To see this, let $W=v f_{1} v_{1} \cdots f_{\ell} u$ and $W^{\prime}=v f_{1}^{\prime} v_{1}^{\prime} \cdots f_{m}^{\prime} u$ be two walks from $v$ to $u$. If $G$ is bipartite, then $\ell$ and $m$ must have the same parity. So $X=v f_{1} v_{1} \cdots f_{\ell} u f_{m}^{\prime} v_{m-1}^{\prime} \cdots f_{1}^{\prime} v$ is a closed walk of even length. Since $\phi_{w}(X)=0$, we immediately get that $\phi_{w}(W)=\phi_{w}\left(W^{\prime}\right)$. If $G$ is not bipartite, one of the closed walks $v f_{1} v_{1} \cdots f_{\ell} u f_{m}^{\prime} v_{m-1}^{\prime} \cdots f_{1}^{\prime} v$ and $v f_{1} v_{1} \cdots f_{\ell} u e_{1} u_{1} \cdots e_{2 k-1} u f_{m}^{\prime} v_{m-1}^{\prime} \cdots f_{1}^{\prime} v$ is of even length, the cycle $C=u e_{1} u_{1} \cdots e_{2 k-1} u$ being of odd length. Denote this walk of even length by $Y$. By assumption we have $\phi_{w}(Y)=0$. Using the definition of $w^{\prime}(u)$ and (2) we reach the desired conclusion.

Finally, we prove that the edge-weighting $w$ is indeed induced by the vertex-weighting $w^{\prime}$ defined by (2). To see this, let $e=x y$ be an edge in $G$ and $W=y f_{1} v_{1} \cdots f_{\ell} u$ a walk from $y$ to $u$. Then $W^{\prime}=x e y f_{1} v_{1} \cdots f_{\ell} u$ is a walk from $x$ to $u$, and $\phi_{w}\left(W^{\prime}\right)=w(e)-\phi_{w}(W)$. If $\ell$ is even, then by (2),

$$
w^{\prime}(x)+w^{\prime}(y)=2 \phi_{w}\left(W^{\prime}\right)-w^{\prime}(u)+2 \phi_{w}(W)+w^{\prime}(u)=2 w(e) .
$$

The same is true if $\ell$ is odd. This completes the proof of the theorem.

Next we characterize positive-induced edge-weightings. We omit the proof, which goes along similar lines to the proof of Theorem 6 . 
Theorem 7. Let $G=(V, E)$ be a connected graph with an induced edgeweighting $w$. If $G$ is nonbipartite, then $w$ is positive-induced if and only if for every closed walk $W=v_{0} e_{1} v_{1} \cdots e_{2 k-1} v_{2 k-1}\left(=v_{0}\right)$ of odd length in $G$ we have $\phi_{w}(W)>0$. If $G$ is bipartite, then $w$ is positive-induced if and only if for every walk $W=v_{0} e_{1} v_{1} \cdots e_{2 k-1} v_{2 k-1}$ of odd length in $G$ we have $\phi_{w}(W)>0$.

It is obvious that in order to characterize nonnegative-induced edgeweightings, we need only replace the condition $\phi_{w}(W)>0$ in Theorem 7 by the condition $\phi_{w}(W) \geq 0$.

It follows from the proof of Theorem 6 that all induced edge-weightings on a connected graph $G=(V, E)$ (including ones with negative values) can be obtained by assigning arbitrary weights to the edges of a spanning tree (if $G$ is bipartite) or to the edges of a connected spanning unicyclic subgraph whose cycle is odd (if $G$ is not bipartite). Thus the dimension of the vector space of induced edge-weightings is $|V|-1$ if $G$ is bipartite, and $|V|$ if $G$ is not bipartite. A natural basis of this vector space can be derived from the vertex-deleted subgraphs of $G$. Each such subgraph $G-v$ can be identified with the edge-weighting $w_{v}$ defined by

$$
w_{v}(e)= \begin{cases}1, & \text { if } e \in E(G-v), \\ 0, & \text { otherwise }\end{cases}
$$

and this edge-weighting $w_{v}$ is induced by the vertex-weighting $w_{v}^{\prime}$ given by

$$
w_{v}^{\prime}(u)=1 \quad(u \neq v), \quad w_{v}^{\prime}(v)=-1 .
$$

Because the vertex-weightings $w_{v}^{\prime}, v \in V(G)$, are linearly independent, they generate the space of all vertex-weightings. It follows that the edgeweightings $w_{v}, v \in V(G)$, generate the space of all induced edge-weightings; thus, when $G$ is nonbipartite, they form a basis. In the bipartite case, any $|V|-1$ of them constitute a basis.

If $G=(V, E)$ is a graph with an edge-weighting $w$, then Theorems 6 and 7 give necessary and sufficient conditions for $w$ to be induced and positive-induced, respectively. They do not, however, yield polynomial-time recognition algorithms. For this, it suffices to grow a breadth-first search tree $T$ rooted at an arbitrary vertex $u$ of $G$. A vertex-weighting $w^{\prime}$ can then be defined in terms of the edge-weighting $w$ and the weight $w^{\prime}(u)$ of $u$ as in (2), using the paths in $T$ from each vertex $v$ to $u$. It remains to verify 
equation (1) for each edge $x y$ in $E(G) \backslash E(T)$. We remark that if $G$ is nonbipartite and $x$ and $y$ are at the same level of $T$, this equation can be solved for $w^{\prime}(u)$. The value of $w^{\prime}(u)$ should, of course, be the same for all such edges $x y$. This recognition algorithm runs in time linear in the number of edges.

\section{References}

[1] B. Bollobás and A.D. Scott, A proof of a conjecture of Bondy concerning paths in weighted digraphs, J. Combin. Theory (B) 66 (1996) 283-292.

[2] J.A. Bondy, Basic graph theory: paths and circuits, in: R.L. Graham, M. Grötschel and L. Lovász, eds., Handbook of Combinatorics (North-Holland, Amsterdam, 1995) 3-110.

[3] J.A. Bondy and G. Fan, Optimal paths and cycles in weighted graphs, Annals of Discrete Math. 41 (1989) 53-69.

[4] J.A. Bondy and G. Fan, Cycles in weighted graphs, Combinatorica 11 (1991) 191-205.

[5] J.A. Bondy and S.C. Locke, Relative lengths of paths and cycles in 3-connected graphs, Discrete Math. 33 (1981) 111-122.

[6] J.A. Bondy and U.S.R. Murty, Graph Theory with Applications (Macmillan, London and Elsevier, New York, 1976).

[7] G.A. Dirac, Some theorems on abstract graphs, Proc. London Math. Soc. (3) 2 (1952) 69-81.

[8] L. Pósa, On the circuits of finite graphs. Magyar Tud. Akad. Mat. Kutató Int. Közl. 8 (1963) 355-361.

[9] T. Spencer (Personal communication, 1992).

[10] Yan Lirong (Personal communication, 1990).

Received 27 June 2000

Revised 22 May 2001 